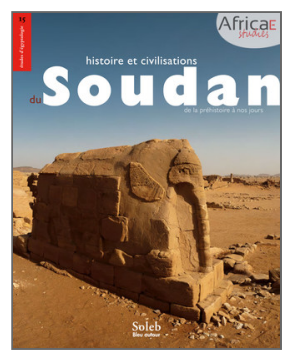

Olivier Cabon (dir.)

Histoire et civilisation du Soudan

De la préhistoire à nos jours

\title{
Un siècle de fouilles archéologiques au Soudan
}

\section{Marc Maillot}

DOI : 10.4000/books.africae. 2847

Éditeur : Africae, Soleb, Bleu autour

Lieu d'édition : Paris, Khartoum

Année d'édition : 2017

Date de mise en ligne : 17 janvier 2022

Collection : Africae Studies

EAN électronique : 9782493207074

\section{OpenEdition}

\section{Books}

http://books.openedition.org

\section{Référence électronique}

MAILLOT, Marc. Un siècle de fouilles archéologiques au Soudan In : Histoire et civilisation du Soudan : De la préhistoire à nos jours [en ligne]. Paris, Khartoum : Africae, 2017 (généré le 28 janvier 2022). Disponible sur Internet : <http://books.openedition.org/africae/2847>. ISBN : 9782493207074. DOI : https:// doi.org/10.4000/books.africae.2847. 
Cette brève historiographie, qui s'appuie entre autres sur les travaux de S.el-Din M.Ahmed ${ }^{1}$, D. Welsby ${ }^{2}$ et D. N. Edwards ${ }^{3}$, nous permettra d'apprécier l'évolution des problématiques de recherches en archéologie soudanaise $\mathrm{du} \mathrm{XIX}^{\mathrm{e}}$ siècle à nos jours, et de réinsérer la politique scientifique française et internationale au sein d'une fresque historique compréhensible. Ce propos servira à encadrer et introduire notre synthèse concernant l'urbanisme méroïtique, rejeton naturel et pourtant assez tardif de la recherche en archéologie soudanaise.

\section{Les récits des grands voyageurs}

le Soudan

\section{8}

histoire et

civilisations
Il faut attendre la fin du XVIII ${ }^{\mathrm{e}}$ et surtout le XIX ${ }^{\mathrm{e}}$ siècle pour que des voyageurs européens partent à la découverte de Méroé, avec pour but, souvent premier, l'exploration des sources $\mathrm{du} \mathrm{Nil}^{4}$. Ces missions, essentiellement fondées sur la lecture des sources classiques, représentent des témoignages précieux des premières observations de sites archéologiques, nous permettant avec le recul de percevoir l'évolution des missions d'exploration autorisant les pillages vers des missions de recherche archéologique ${ }^{5}$.

Le précurseur, James Bruce (1730-1794), entreprit une mission à partir de 1769 de recherche des sources du Nil qu'il situait en Abyssinie ${ }^{6}$. Après avoir effectué un voyage le menant de Massaouah, sur la rive de la mer Rouge, à Gondar, capitale de l'Éthiopie, puis le long du Nil Bleu jusqu'au lac Tana, sa source, il entreprit un voyage de retour vers l'Égypte. C'est lors de ce trajet, accompagné d'un dessinateur italien nommé Belaguni, qu'il observa le 21 octobre 1772 les premières ruines depuis Axoum, près du village de Begrawiya. Le récit de son exploration, Voyage aux sources du Nil, en Nubie et en Abyssinie, pendant les années 1768-1773, premier ouvrage à associer les vestiges découverts à l'antique capitale de Méroé, fut publié en 1790.

Ce n'est qu'au début du XIx ${ }^{\mathrm{e}}$ siècle que cet ensemble de ruines est à nouveau repéré par Johan-Ludwig Burckhardt (1784-1817), envoyé par l'African Society de Londres pour une mission d'exploration de l'Afrique. Se rendant à Shendi, il observa en avril 1814 des fondations de briques rouges, "plus grossières que celle utilisées en Égypte», et quelques constructions en pierre sur une surface assez étendue ${ }^{7}$.

Le Nantais Frédéric Cailliaud (1787-1869) se joignit à l'expédition militaire menée, à partir de 1820, par Ismaël Pacha, troisième fils du vice-roi d'Égypte Méhémet Ali, dans l'objectif de découvrir la cité antique de Méroé $^{8}$. Remontant le Nil, il repéra et étudia différents sites nubiens d'importance, tels que la nécropole de Sedeinga, le temple de Soleb (figure 1), Kerma, la nécropole de Nouri et le Gébel Barkal ${ }^{9}$. Précédant 


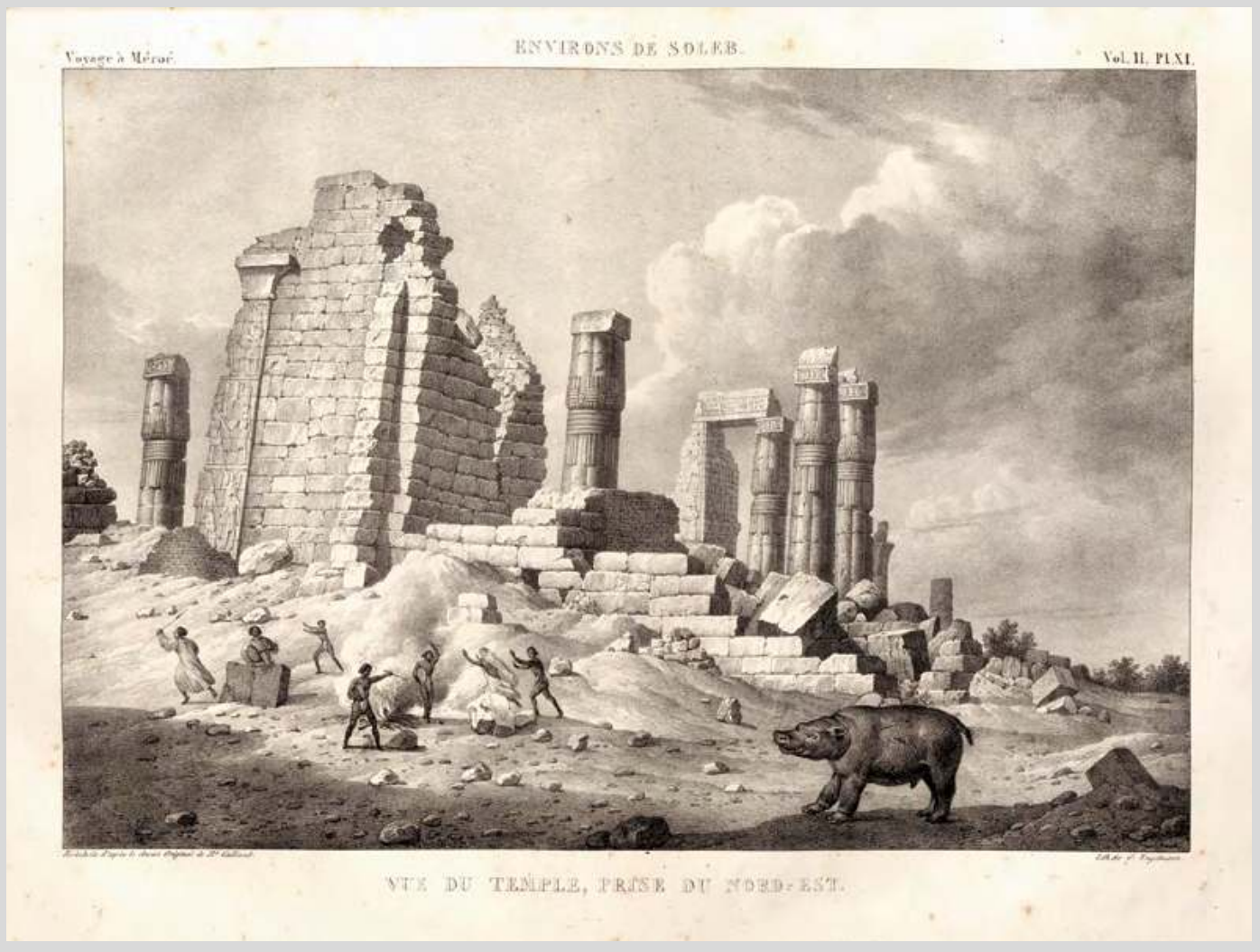

Figure 1: Temple de Soleb, vue du nord-est (d'après Cailliaud 1826, vol. II, pl. XI).

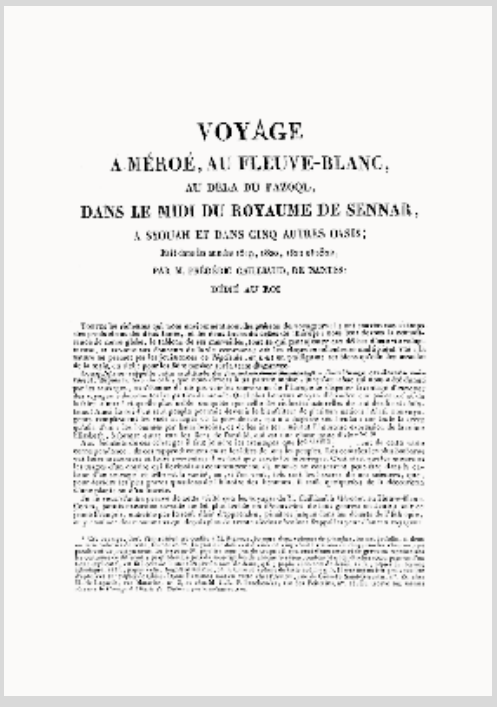

Figure 2: Première page du Voyage à Méroé et au Fleuve Blanc au-delà du Fàzoql (d'après Cailliaud 1826).

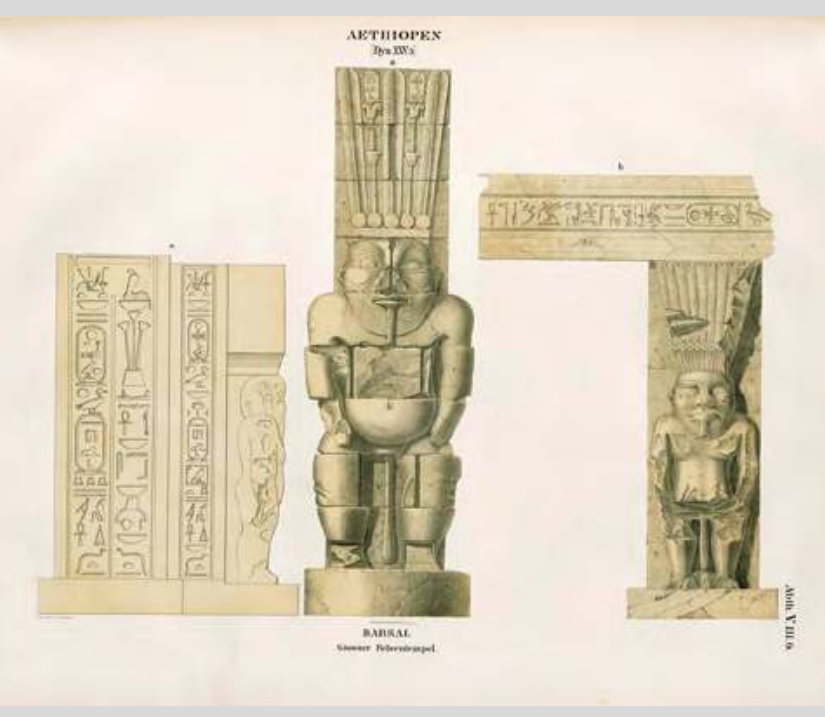

Figure 3 : Barkal Felsentempel, Denkmäler aus Aegypten und Aethiopien, Tafel Werke, Band X, Aethiopien, Abth V. B1.6. (d'après http://edoc3.bibliothek.uni-halle.de/lepsius/ tafelwas.html) (C) ULB Halle/Lepsius-Projekt. un siècle de fouilles 
le Soudan

450

histoire et

civilisations l'armée en direction de Shendi avec son compagnon de voyage PierreConstant Letorzec, il aperçut les pyramides de Begrawiya le 25 avril 1821, puis découvrit la ville de Méroé. Ainsi, son ouvrage Voyage à Méroé, au Fleuve Blanc, publié en 1826, représente la première étude archéologique du site, richement illustrée de ses dessins (figure 2$)^{10}$.

Quelques mois seulement après la visite des sites par Cailliaud, Linant de Bellefonds (1799-1883), mandaté par William Bankes pour explorer la Nubie et voyageant dans la région de Shendi afin de se rendre à Sennar, passa à proximité du site en novembre 1821, sans s'y arrêter. Il retourna en revanche étudier les pyramides de Méroé en avril 1822, un an après Cailliaud, après avoir découvert pour la première fois les ruines de Naga et Musawwarat es-Sofra ${ }^{11}$. (Voir p. 209, 210 et 217.)

La publication des découvertes de ces voyageurs, notamment celle des pyramides de Méroé, éveilla rapidement l'intérêt de "voyageurspilleurs» tels que Giuseppe Ferlini $(1800-1870)^{12}$. Ayant démantelé plusieurs pyramides en 1834, il fit exploser l'imposante pyramide Beg. N6, mettant ainsi au jour le trésor de la candace Amanishakheto qu'il vendit au musée de Munich en 1839 et au musée de Berlin en 1844. Ce n'est qu'avec l'expédition prussienne de Carl Richard Lepsius (1810-1884), entre 1842 et 1845 , que l'objectif d'une étude archéologique poussée se développe ${ }^{13}$. Ainsi les Denkmäler aus Aegypten und Aethiopien (figure 3) représentent la première référence bibliographique dans le relevé et l'étude des sites archéologiques nubiens, tels que la nécropole de Méroé et les sites de Musawwarat es-Sofra et Naga, mettant en valeur l'importance archéologique de la civilisation méroïtique ${ }^{14}$.

Insistons sur le rôle joué par C. R. Lepsius en 1842, où ce dernier parcourt la vallée du Nil en Égypte, puis va remonter le fleuve jusqu'au cœur du Soudan. J.-F. Champollion était pourtant venu vingt ans auparavant avec une expédition envoyée par le roi de France mais s'était arrêté à la deuxième cataracte. Lepsius descend alors jusqu'à Méroé, en collectant les artefacts qui jalonnent son passage, expliquant ainsi pourquoi le musée de Berlin est le plus riche d'Europe en antiquités soudanaises.

En 1845, ces artefacts parviennent à Hambourg. La publication des Denkmäler aus Aegypten und Aethiopien, «Monuments d'Égypte et de Nubie», va permettre de redécouvrir la région et de présenter de nombreux textes, dessins et fac-similés. Lepsius, contrairement à Cailliaud, est égyptologue de formation; ces Denkmäler constituent une somme remarquable d'informations doublée d'un traitement scientifique de grande qualité. 


\section{Prémices de l'archéologie méroïtique fin du XIX siècle-premier quart du XX $X^{e}$ siècle}

\section{La région de Méroé}

Les premières interventions archéologiques sur des sites en territoire soudanais ont été menées par W. Budge suite à la conquête britannique du Soudan en 1898. Son programme de fouille des sites de Méroé et du Gébel Barkal a majoritairement consisté en la récolte du matériel archéologique ${ }^{15}$. Une prospection archéologique des sites méroïtiques de l'̂̂le de Méroé, dans le Boutana, fut conduite par J. W. Crowfoot en 1906. Premier à réaliser un travail archéologique novateur, à la fois analytique et synthétique, il entreprend notamment une étude des ressources économiques et une typologie des sites archéologiques de la région ${ }^{16}$. Deux ans plus tard, une description des vestiges de Naga et Musawwarat es-Sofra fut réalisée en 1908 par P. D. Scott-Moncrief ${ }^{17}$.

Le premier programme de fouille de la ville de Méroé, menée par la mission de l'université de Liverpool, fut dirigé par J. Garstang de 1909 à $1914{ }^{18}$. Les fouilles furent vouées à l'étude de la "cité royale», de grands monuments adjacents et des nécropoles populaires orientales. Les travaux de J. Garstang sont précurseurs dans la tentative de reconstitution de l'histoire architecturale de la ville, fondée sur la diversité des matériaux de construction, ainsi que dans l'établissement d'une première typologie de la céramique usuelle.

\section{La Basse-Nubie}

Les premières opérations archéologiques d'envergure en Nubie résultent de la décision d'agrandissement du premier barrage d'Assouan par l'Égypte de 1908 à 1910. Ainsi, la First Archaeological Survey of Nubia, première prospection en Nubie égyptienne, fut destinée à sauver les sites menacés d'immersion. Se déroulant durant quatre saisons, de 1907 à 1911, elle couvrit $152 \mathrm{~km}$ de la vallée du Nil au sud de la première cataracte, entre Shellal et Ouadi es-Seboua.

Dirigée d'abord par G. A. Reisner, puis par Colin Firth, elle représente la fouille de 151 cimetières, soit 8000 tombes individuelles, et de seulement 6 sites d'habitat superficiellement explorés ${ }^{19}$. Parmi le groupe W, correspondant aux vestiges gréco-romains et méroïtiques, l'on ne recensa que des tombes, mises au jour dans la partie méridionale de la Basse-Nubie. Ce survey, dont le travail s'est centré sur la distinction des différentes cultures «nubiennes» et leurs contacts avec l'Égypte prédynastique et pharaonique, a été rapidement complété par d'autres expéditions aux abords de la frontière entre l'Égypte et la Basse-Nubie. 
En Nubie soudanaise, L. Woolley et R. D. MacIver furent les premiers à entreprendre un programme de fouille d'une ville méroïtique ${ }^{20}$ et de son cimetière associé21 sur le site de Karanóg (figure 4). Cette fouille de la mission de l'université de Pennsylvanie, en 1909, a permis de documenter l'architecture civile méroïtique dans une région encore non étudiée et de décrire un matériel archéologique qui se distingue de celui provenant des sites de la région du Boutana.

De 1910 à 1912, les fouilles de la ville de Faras, dirigées par F.Ll.Griffith ${ }^{22}$, de l'université d'Oxford, ont mis au jour une structure d'importance considérée comme un exemple d'architecture civile méroïtique: le «palais occidental» (figure 5). Dans le domaine funéraire, le vaste cimetière associé à la ville contenait un matériel archéologique remarquable.

\section{Les marges méridionales du royaume}

Durant la même période, la mission de la Wellcome Foundation fouilla en 1914, sous la direction d'O.G. S. Crawford, le village d'Abou Geili ${ }^{23}$ situé sur le Nil bleu à une latitude très légèrement supérieure à celle de Kosti, ainsi que, de 1909 à 1914, sous la direction de Fr. Addison, le cimetière du Gébel Moya ${ }^{24}$ situé dans les terres, entre Abou Geili et Kosti. Leur position très au sud par rapport au royaume de Méroé et la particularité du matériel mis au jour rendent ardue l'association de ces sites à la période et aux cultures kouchites.

\section{L'entre-deux-guerres}

histoire et civilisations

\section{Les nécropoles royales et les grands centres urbains de Napata et Méroé}

Les missions d'envergure de l'université de Harvard et du musée des Beaux-Arts de Boston aux nécropoles royales d'el-Kourrou et Nouri, ainsi que dans les centres urbains de Napata (1916-1919) et Méroé (1922-1925), sont une étape majeure dans la connaissance historique et chronologique de l'époque koushite.

Les deux nécropoles d'el-Kourrou et Nouri, à proximité du Gébel Barkal, fouillées par l'expédition Harvard-Boston sous la direction de G.A. Reisner, ont révélé les tombes des souverains de la $\mathrm{XXV}^{\mathrm{e}}$ dynastie, de leurs prédécesseurs et de leurs successeurs napatéens, et leur abondant matériel associé ${ }^{25}$. Au Gébel Barkal, ce sont les temples et palais pharaoniques et kouchites de Napata qui sont fouillés ${ }^{26}$, ainsi que les deux groupes de pyramides situées à l'arrière nord-ouest du Gébel, pouvant être datés du Méroïtique ancien ${ }^{27}$. À Méroé, le travail consista en la fouille des trois nécropoles à pyramides, les cimetières nord, ouest et sud 


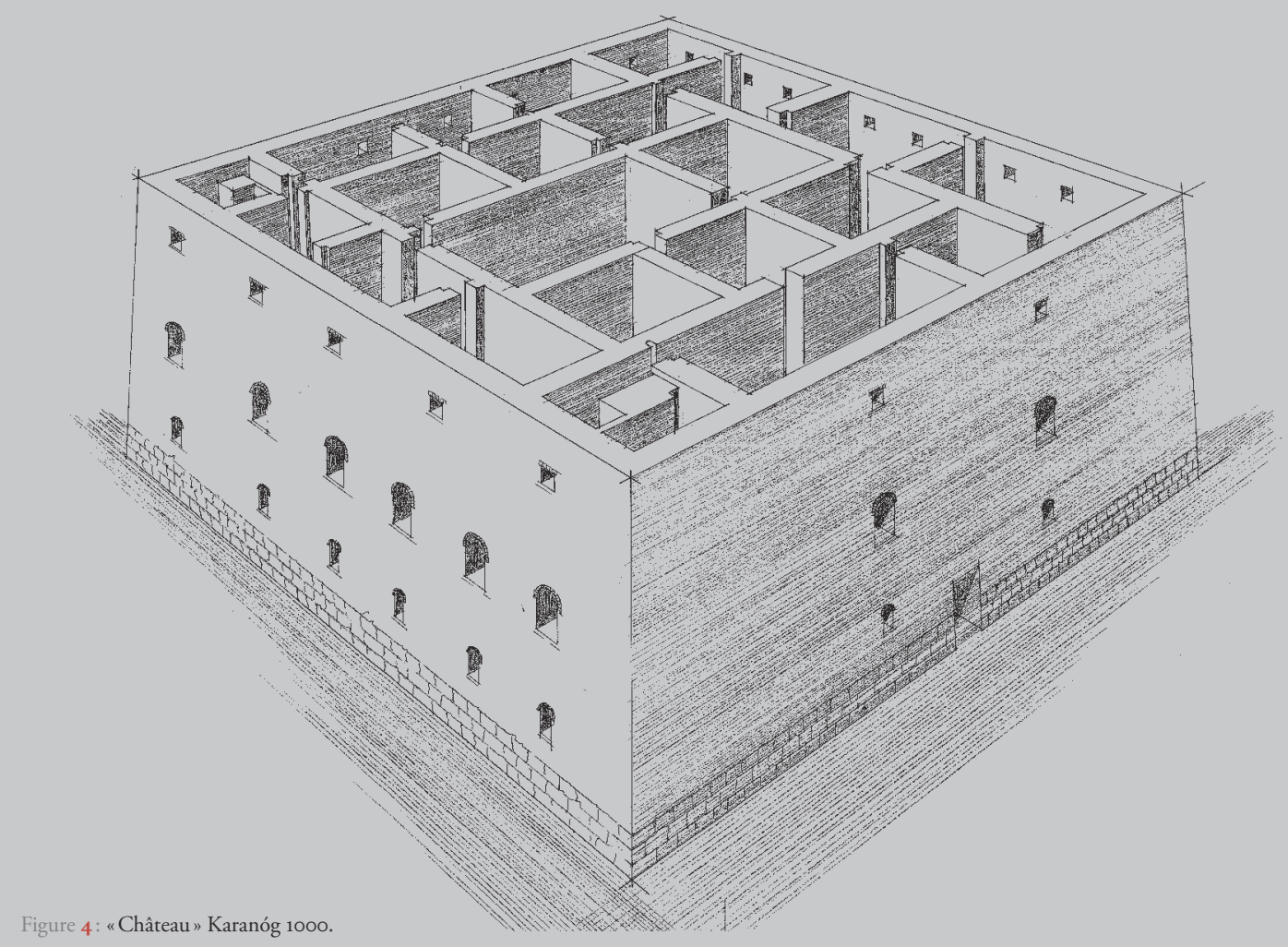

Figure 4: « Château » Karanóg 1000.

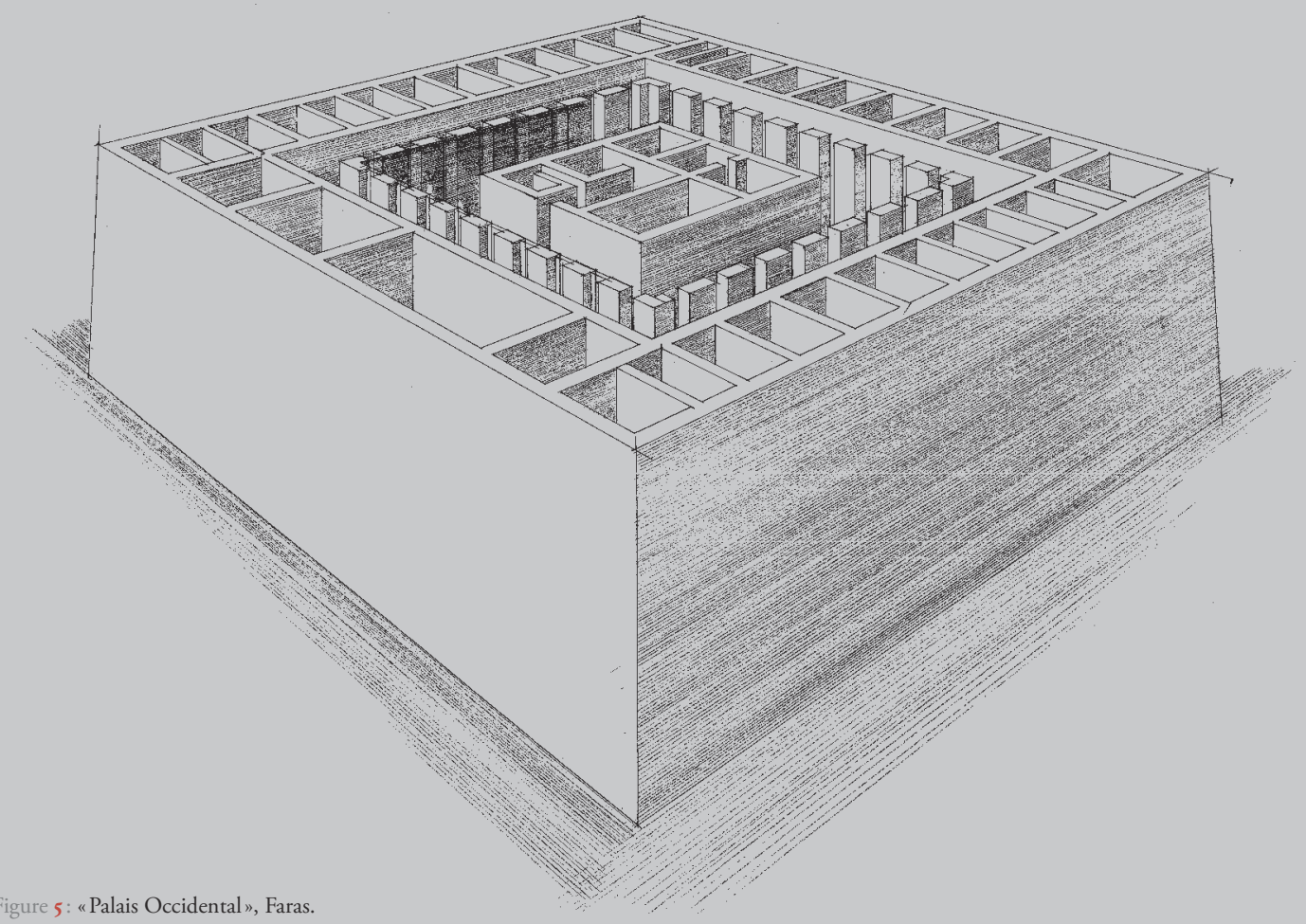


le Soudan

histoire et civilisations

de Méroé ${ }^{28}$. L'ensemble de ces fouilles, fondées sur l'étude sérielle des tombes royales napato-méroïtiques et de leurs inscriptions, a permis à G. A. Reisner d'établir la première chronologie de la succession royale kouchite ${ }^{29}$.

\section{La Basse-Nubie}

En parallèle à ces missions, la couverture des sites méroïtiques du Boutana, dont Naga, Musawwarat es-Sofra, Basa ou le Gébel Geili et de leurs nombreux vestiges monumentaux a été assurée par J.W. Crowfoot, F. A. Addison, D. Dunham et G. O. Whitehead ${ }^{30}$. Ce travail a permis de confirmer l'importance de l'«île de Méroé» mentionnée par les auteurs anciens.

Suite à une extension du premier barrage d'Assouan, une nouvelle montée des eaux fut à l'origine de la mise en ouvre du Second Archaeological Survey of Nubia de 1929 à 1934, sous la direction de W. B. Emery ${ }^{31}$. La prospection, s'étendant au sud jusqu'à la frontière soudanaise, confirma la rareté des vestiges méroïtiques en Basse-Nubie. Les vestiges d'habitat au Wadi el-Arab comprennent plusieurs maisons et une presse à vin, dont l'ensemble peut être daté à partir du $\mathrm{III}^{\mathrm{e}}$ siècle apr. J.-C. ${ }^{32}$

Enfin, durant la même période, la mission de l'université d'Oxford, sous la direction de M. F. L. Macadam, fouilla le centre urbain de Kawa, se concentrant sur l'architecture du temple et l'étude de ses inscriptions, mais mettant également au jour plusieurs habitations napatéennes et méroïtiques associées aux sanctuaires ${ }^{33}$.

\section{Les années 1950 à 1970}

\section{sauvetage en Nubie et retour à Méroé}

\section{Le Boutana occidental}

Durant les années 1950, deux prospections de faible ampleur ont été menées dans le Boutana par H.S.Smith et A.Adam ${ }^{34}$, ainsi que par H.G. BalfourPaul ${ }^{35}$. Le premier survey a révélé quatre sites probablement méroïtiques ou antérieurs, dont le Gébel Sirefāt, son hafir et son ensemble de bâtiments de maçonnerie similaire à celle de Musawwarat es-Sofra.

Dans le Kéraba, la partie nord-ouest de l'«île de Méroé», l'université Humboldt réalisa une prospection en 1957-1958, dirigée par Fr. Hintze ${ }^{36}$. Ce survey a consisté en un relevé des coordonnées des sites archéologiques, dont Naga, Moutmir, Basa ou Awalib, le ramassage du matériel de surface et le relevé d'inscriptions. Parallèlement, Musawwarat es-Sofra a fait l'objet d'opérations de fouille massives sous la direction de Fr. Hintze, tendant à distinguer les différentes composantes du Grand Enclos, afin de pouvoir établir une chronologie relative du site, à partir de sondages ${ }^{37}$. 
Dans la vallée, J. Vercoutter et Thabit Hassan Thabit, à la demande du service des Antiquités du Soudan, entreprirent de 1958 à 1960 des fouilles dans le centre urbain d'Ouad Ben Naga ${ }^{38}$. Celles-ci comprirent notamment la fouille partielle d'un palais daté du $\mathrm{I}^{\mathrm{er}}$ siècle av. J.-C., ainsi que le sondage ou la fouille partielle de deux petits temples, dont un dédié à Isis, et de plusieurs bâtiments administratifs ou religieux.

Les fouilles de la ville de Méroé sont reprises au milieu des années soixante par l'université de Calgary, sous la direction de P. L. Shinnie ${ }^{39}$. Le but de ces travaux d'envergure est alors d'effectuer des fouilles stratigraphiques destinées à réaliser une séquence chronologique complète de la ville, dont les premières occupations dans la zone d'habitat nord peuvent être datées du $\mathrm{X}^{\mathrm{e}}$ siècle av. J.-C. Ces recherches englobent non seulement l'architecture religieuse monumentale (temple d'Amon et chapelles de la voie sacrée) mais aussi les occupations domestiques. Une étude sur la métallurgie du fer, la production céramique et les conditions écologiques du site permettent de compléter la connaissance des différentes phases culturelles du site.

\section{La Basse-Nubie et la campagne de l'Unesco}

De la construction du haut barrage d'Assouan a résulté, de 1959 à 1969, la "campagne de sauvegarde des monuments de Nubie», dirigée par l'Unesco. Outre le déplacement des grands temples de Nubie du côté égyptien (Abou Simbel et Philae) (figure 6), cette campagne a consisté en une vaste opération internationale de prospection systématique et de fouille s'étendant jusqu’à l'extrémité sud de la région du Batn el-Haggar, entre les seconde et troisième cataractes. Cette fouille a permis entre autres le repérage de 1000 sites archéologiques sur $160 \mathrm{~km}$ de la vallée du Nil. Bien que l'ensemble des résultats n'ait pas été publié, les nombreux rapports de fouille, révélant de nombreux sites méroïtiques, ont offert une meilleure compréhension de l'archéologie dans cette région et de son histoire culturelle ${ }^{40}$.

En Nubie égyptienne, les sites de Qasr Ibrim ${ }^{41}$, Tômass2, Gébel Adda ${ }^{43}$ et Ash-Shaukan ${ }^{44}$ ont livré une riche documentation sur l'organisation de l'occupation méroïtique à l'extrême nord du royaume. Des vestiges de la même période ont également été mis au jour à Arminna-Ouest par l'université de Pennsylvanie ${ }^{45}$ et à Gézira Dabarosa par l'université du Colorado $^{46}$. La mission du service des Antiquités du Soudan, quant à elle, a mis au jour des sites d'habitat à Faras ${ }^{47}$ et sur les îles de Meinarti ${ }^{48}$, de Gaminarti et de Meili ${ }^{49}$.

L'ensemble de ces travaux, plus détaillés que ceux réalisés en Nubie égyptienne, et la mise en eau de l'ensemble de cette région sous le Lac Nubia, ont mené les chercheurs à la rédaction de synthèses généralistes 
sur l'état des recherches après cette campagne de prospection, comme les ouvrages Egypt in Nubia de W. B. Emery, publié en 1965, et Nubia under the Pharaohs de Br. Trigger, publié en $1976^{50}$. Enfin, l'important Nubia, Corridor to Africa de W.Y.Adams, publié en 1977, fournit une synthèse des résultats de l'ensemble des campagnes de prospections de la Nubie, s'intéressant aux relations particulières entre la Basse-Nubie et l'Égypte mais faisant également le point sur les connaissances archéologiques du reste du Nil Moyen ${ }^{51}$.

Plus au sud, sur l'île d'Argo, Tabo a été fouillé par la mission de la fondation H. M. Blackmer et l'université de Genève, sous la direction de Ch. Maystre ${ }^{52}$, révélant un temple construit à la XXV dynastie, utilisé et remanié jusqu’à l'époque méroïtique.

\section{Les fouilles récentes}

\section{vers un renouveau des problématiques?}

le Soudan

\section{6}

histoire et civilisations
Suite à la disparition de la région de la Basse-Nubie, la recherche archéologique s'est trouvée contrainte de se tourner vers les territoires méridionaux. L'intérêt pour les grands établissements urbains de l' «île de Méroé» a perduré, se reflétant notamment dans l'ouvrage The Kingdom of Kush de D.A. Welsby, publié en 1996, synthèse des grandes avancées sur la connaissance de l'archéologie et l'histoire de la période koushite, ou encore dans la synthèse de L. Török, The Kingdom of Kush: Handbook of the NapatanMeroitic Civilization, publié en $1997^{53}$.

\section{Continuité des prospections}

Durant les années 1990 se développe la conscience de la nécessité de continuer les prospections de terrain en prévision des destructions consécutives à la construction d'un réseau viaire ${ }^{54}$ ou de barrages hydrauliques ${ }^{55}$ d'une part, mais également du développement urbain d'autre part ${ }^{56}$. Et l'on débute avec la prospection d'aires géographiques encore méconnues comme le Nil Blanc ou les régions désertiques ${ }^{57}$. De telles enquêtes mettent en exergue le potentiel d'études plus ambitieuses orientées sur la relation entre les établissements humains et leur environnement.

Ainsi, lors de la prospection le long de la route reliant Begrawiya et Atbara, une vaste nécropole a été découverte à Gabati. Le tracé de la route ne pouvant être déplacé, le site a été fouillé en urgence durant l'hiver 1994-1995, révélant une occupation funéraire allant de la période méroïtique à l'époque médiévale ${ }^{58}$.

Entre 2001 et 2009, le Merowe Dam Archaeological Salvage Project - MDAsp, prospection d'envergure internationale, a été monté afin de mettre au jour et étudier les vestiges menacés par la construction 
du barrage hydroélectrique de Merowe, implanté à une quarantaine de kilomètres en amont du Gébel Barkal. La prospection de la zone inondable a été répartie entre de nombreuses missions archéologiques et a permis de mettre en évidence le rôle important de la région dans l'administration koushite de la quatrième cataracte ${ }^{59}$. Les sites majeurs comprennent des nécropoles méroïtiques au riche matériel funéraire ${ }^{60}$, ainsi que l'île d'Oumm Mouri, un vaste domaine habitable à vocation agricole constitué d'une succession de huttes et de maisons de briques crues ou cuites ou en pierre, datables du méroïtique tardif à la période médiévale ${ }^{61}$.

\section{Fouilles programmées}

Aujourd'hui, d'importants vestiges méroïtiques sont en cours de fouilles sur une dizaine de sites d'occupation méroïtique allant de la région de la Moyenne-Nubie au Nil Blanc. Entre les troisième et quatrième cataractes, l'important site urbain de Doukki Gel, d'une étendue de 8 ha, comprenant un temple et des palais, est fouillé sous la direction de Ch. Bonnet (université de Genève) ${ }^{62}$, tandis que la ville de Kawa est fouillée depuis 1997 sous la direction de D. Welsby (Sudan Archaeological Research Society) ${ }^{63}$. Enfin, au Gébel Barkal, deux missions se partagent les fouilles des nombreux monuments royaux et administratifs: la mission d'E. Ciampini (université de Rome) s'intéresse majoritairement au palais B1500 ainsi qu’à deux autres bâtiments, B 2200 et B $2400^{64}$, et la mission de T. Kendall et el-Hassan Ahmed Mohamed (musée de Boston) étudie pour sa part les nombreux temples du site ${ }^{65}$.

Les sites du Soudan central situés le long de la vallée du Nil sont nombreux à faire l'objet d'un programme de fouille. Près d'Atbara, la NCAm a créé en 2000 le Berber-Abidya Archaeological Project, en collaboration avec le British Museum, qui comprend à ce jour deux chantiers de fouille. Ainsi, à Dangeil, grand centre urbain koushite, le temple d'Amon, construit à l'époque napatéenne et en activité jusqu'à la fin de l'époque méroïtique, est fouillé depuis 2000 sous la codirection de Salah Mohamed Ahmed et J. Anderson (Ncam et British Museum) ${ }^{66}$.

La ville royale de Méroé fait l'objet de deux programmes de fouille, le premier depuis 1999, sous la direction de Kr. Grzymski et Ali Osman Mohamed Saleh (Ontario Royal Museum et université de Khartoum). La politique de fouille est généraliste (prospections pédestres et magnétométriques) et s'intéresse principalement aux structures administratives et à la restauration du grand temple d'Amon de la ville ${ }^{67}$, tandis que la mission dirigée par S. Wolf (German Archaeological Institute) se concentre sur la fouille des «bains royaux» et de leur système hydraulique depuis $1999^{68}$. 


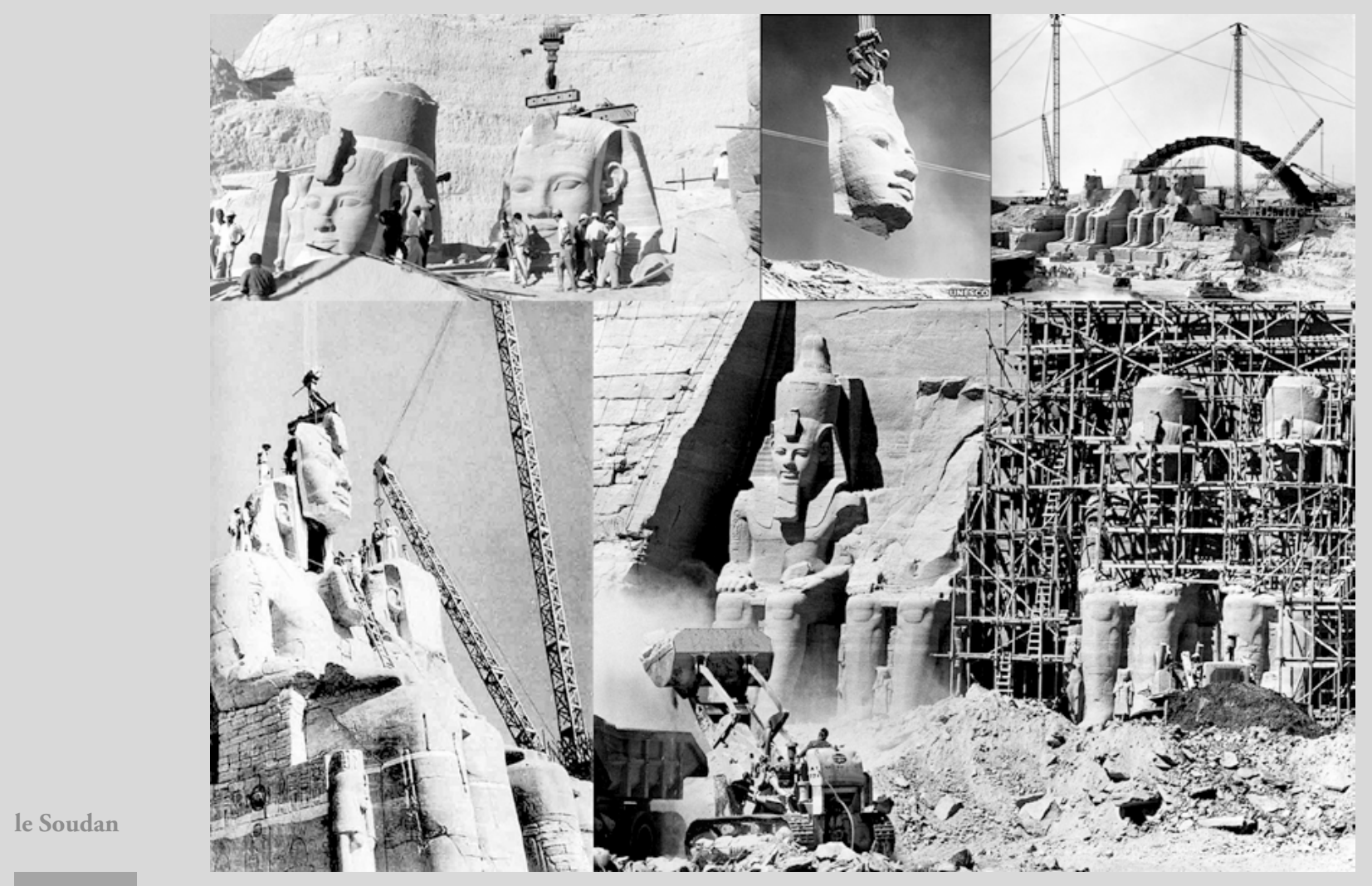

Figure 6: Déplacement des temples de Nubie, campagne de l'Unesco.

histoire et civilisations

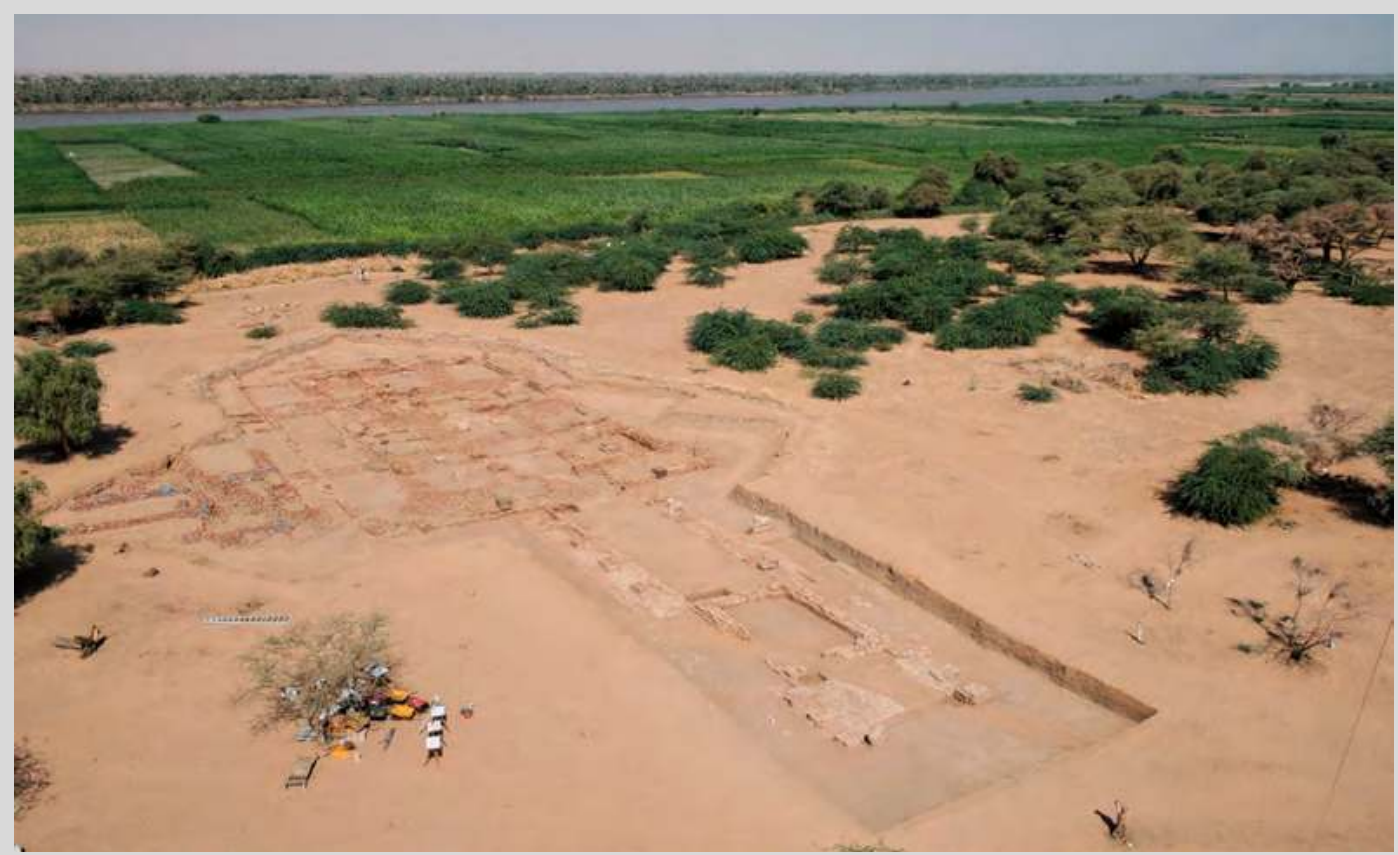

Figure 7: Vue aérienne du temple d'Amon d'el-Hassa. 
Le site urbain d'Hamadab est fouillé depuis 2001 sous la direction de P. Wolf (German Archaeological Institute, université de Shendi), dans un programme alliant magnétométrie et fouille de secteurs (habitats, fours céramiques, enceinte) ${ }^{69}$.

Le site d'Awalib, découvert lors du survey du tracé de la route Begrawyia-Shendi en 1995, est fouillé depuis 2001 sous la direction de H. Paner (musée de Gdańsk, Pologne). Il présente 5 kôms, dont un petit temple et des bâtiments administratifs ${ }^{70}$.

Le site d'el-Hassa (figure 7), prospecté en 2002 et étudié sous la direction de V.Rondot (Sfdas, France) depuis 2004, est une ville méroïtique dont seul le complexe du temple d'Amon a été fouillé de manière approfondie. Le site a également fait l'objet d'une exploration par magnétométrie et de prospections de surface ayant permis d'obtenir une vision d'ensemble de la trame urbaine de la ville ${ }^{71}$. Notons la présence proche du site de Damboya, prospecté mais non fouillé, qui comprend un palais royal et probablement un autre bâtiment de nature indéfinie.

Enfin, le site de Mouweis, fouillé sous la direction de M. Baud puis de M. Millet (musée du Louvre) depuis 2007, adopte une méthodologie plurielle, alliant magnétométrie et prospections pédestres, carottages et sondages, ainsi que la fouille poussée de certains monuments ou de secteurs artisanaux ${ }^{72}$.

Dans le Boutana, les sites majeurs de Musawwarat es-Sofra et Naga continuent à faire l'objet d'études poussées, sous les directions respectives de Cl. Näser (université de Humboldt) depuis $2005^{73}$, de D. Wildung (directeur administratif, musée Égyptien de Berlin) et K. Kröper (directeur du chantier de fouille) depuis $1995^{74}$, consacrées sur le premier site à l'étude des premières phases de construction et d'occupations (napatéennes et méroïtiques anciennes) et à l'étude des temples sur le second site.

Il semble au regard des fouilles actuelles que, tandis que certaines missions s'attachent à fouiller des nécropoles élitaires méroïtiques au fort potentiel pour la compréhension historique et matérielle de l'Empire de Méroé (Saï, Sedeinga ou Berber ${ }^{75}$ ), la majorité des programmes de recherche en cours se concentre autour de la question de l'urbanisme méroïtique.

Plusieurs problématiques peuvent découler de l'étude de sites dits "urbains». Certaines missions tentent de comprendre l'organisation générale et le fonctionnement d'un établissement urbain à l'époque méroïtique (Mouweis, Awalib, Hamadab, Kawa et Doukki Gel). Leur méthodologie est multiple, alliant prospections pédestres et magnétométrie afin d'obtenir un plan général de la sectorisation des sites, carottages et petits sondages servant à évaluer la durée d'occupation des sites, et fouille plus précise des secteurs ou de bâtiments choisis afin de réaliser des études 
le Soudan

460

histoire et civilisations

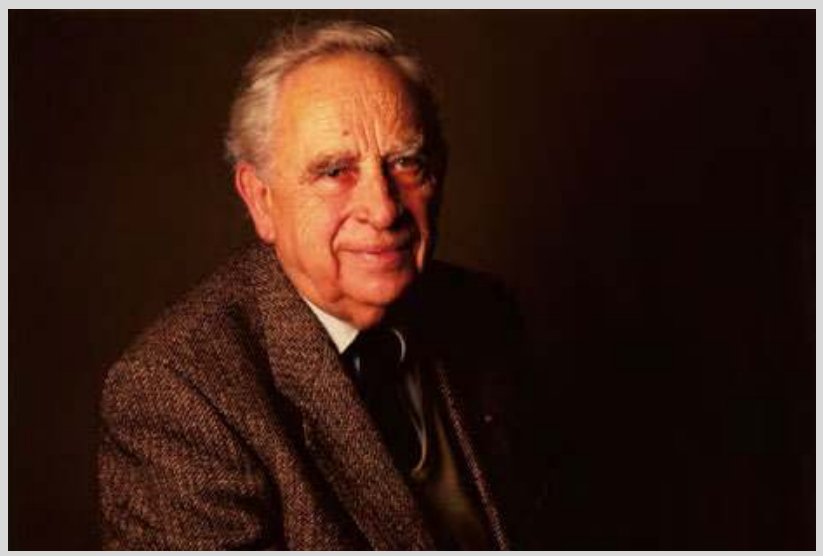

Figure 8: Jean Vercoutter.

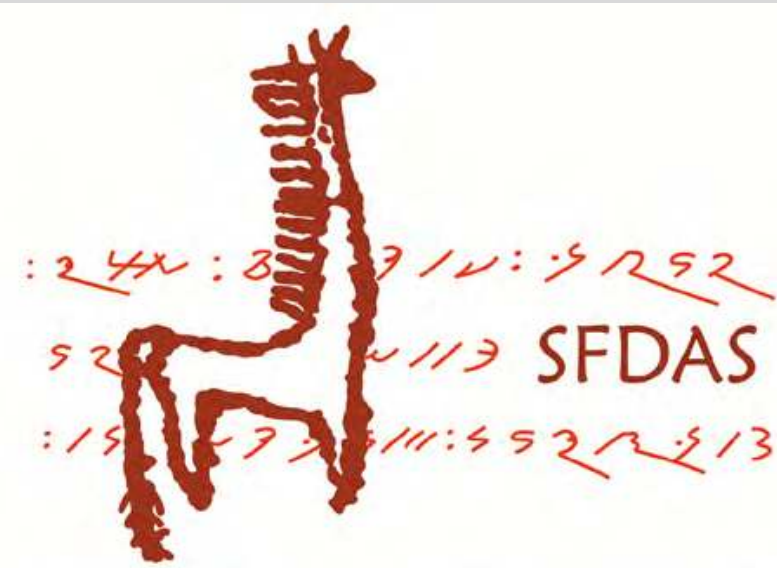

Section française de la direction des antiquités du Soudan

Figure 9: Section française de la direction des Antiquités du Soudan (Sfdas).

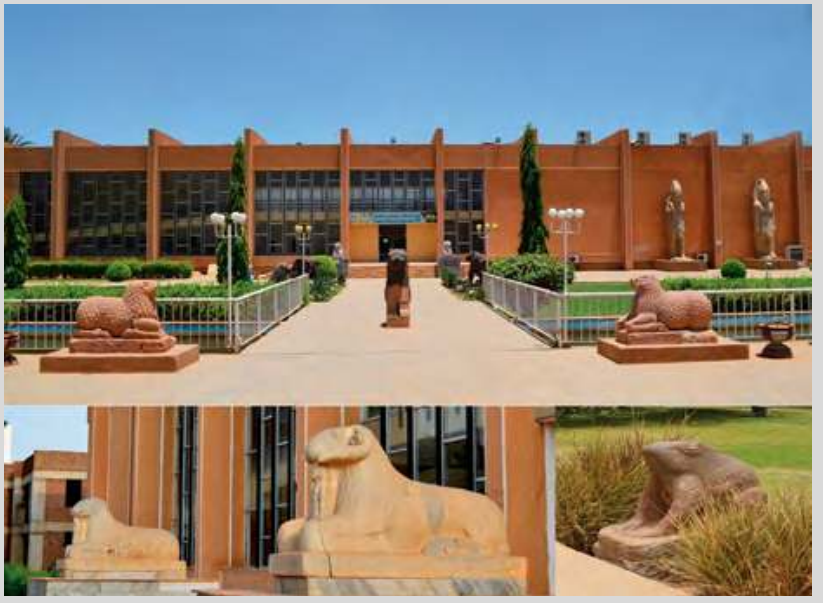

Figure 10: musée national du Soudan, entrée principale. 
de cas: palais (Mouweis) et bâtiments administratifs (Awalib, Doukki Gel), petits temples (Mouweis), aires d'habitat-artisanat ou encore zones d'artisanats du feu avec fours (Kawa, Hamadab, Mouweis).

D'autres missions concentrent leurs recherches autour d'un monument, en étudiant à la fois ses caractéristiques architecturales, son évolution chronologique et ses caractéristiques fonctionnelles. Les questions liées à la royauté, à la religion et à l'administration méroïtique sont au premier plan. Il en va ainsi en majorité de la fouille de temples d'Amon (Naga, elHassa et Dangeil). D'autres missions s'intéressent notamment à l'étude des palais (Gébel Barkal) ou encore de structures associées à la royauté (les «bains royaux» de Méroé, ou les complexes B 2200 et B 2400 de Napata).

Ce rapide résumé présente ainsi un changement important dans les problématiques de fouilles, longtemps concentrées dans la partie septentrionale de l'Empire et plus particulièrement dans le domaine funéraire. Depuis les années 1970, et notamment depuis 1990 avec les fouilles entreprises dans l'île de Méroé, la découverte progressive d'un réseau urbain au maillage dense et complexe nous permet d'entreprendre une étude synthétique centrée sur les composants urbains majeurs (voir chapitre suivant).

\section{La Sfdas et l'archéologie française au Soudan}

Après Lepsius et Ferlini, pendant la Turkiyya, période de l'occupation turcoégyptienne, l'administration coloniale est soutenue par les Occidentaux. Les taxes écrasantes et l'occidentalisation croissante des Turcs entrainent une réaction soudanaise, une révolte contre le pouvoir et l'établissement, pendant une douzaine d'années, d'un pouvoir islamique établi à Omdurman : la Mahdiyya. Le Mahdi meurt en 1885 et son bras droit Abdullahi le remplace. Durant toute cette période, l'archéologie subit un coup d'arrêt.

Placées sous la direction du général Kitchener entre 1896 et 1898 , les troupes anglaises parachèvent leur conquête du Soudan lors de la bataille d'Omdourman. Kitchener instaure un nouveau pouvoir colonial, le "condominium anglo-égyptien». Les archéologues reviennent: ils sont essentiellement britanniques, puis allemands. Les Français, absorbés par l'Égypte à la suite des découvertes de Champollion et en lutte avec les Anglais pour la constitution d'un Empire colonial attendront plus longtemps. Entre 1898 et 1956, l'administration britannique est la première à développer la gestion du patrimoine archéologique soudanais. En 1939, un poste spécifique est créé, celui de Commissioner for Archaeology, assuré en premier lieu par A. J. Arkell ${ }^{76}$. Les Britanniques mettent également en œuvre, en 1905, la première législation sur les antiquités, à la base de la loi actuelle. 
Peu après la proclamation de l'indépendance, le 19 décembre 1955, les autorités soudanaises confient à un Français, Jean Vercoutter (figure 8), la direction de la gestion des antiquités. C'est lui qui assurera la transition menant à la création d'un service soudanais spécialisé. Homme d'expérience au Soudan, il fouille dès 1953 la ville fortifiée du Moyen Empire à Kor, en Basse Nubie, et lance en 1954 les premiers travaux sur l'île de Saï. J.Vercoutter est également très impliqué dans le sauvetage des antiquités menacées par le barrage d'Assouan ${ }^{77}$. Il supervise plusieurs missions de prospection des zones en danger, la couverture photographique associée, et fait appel à la communauté internationale pour intégrer le Soudan à la grande campagne Unesco. Nommé professeur à l'université Charles-de-Gaulle-Lille 3 en 1961, il engage l'institution dans la campagne de sauvetage des monuments de la Nubie. À l'époque, cette implication fera de l'université le centre névralgique de la recherche française en archéologie soudanaise $^{78}$, via l'association d'une unité CNRS à l'institution et le lancement de deux fouilles archéologiques sur les sites d'Akcha et de Mirgissa ${ }^{79}$. En 1969, la fin de la campagne de Nubie permet à J. Vercoutter de concentrer ses travaux à Saï, mission qu'il dirigera jusqu’en 1981.

Dans le même temps, la section française de la direction des Antiquités du Soudan (Sfdas) ${ }^{80}$ est fondée en 1967, toujours à l'initiative de Vercoutter (figure 9). Régie par «l'Accord de coopération culturelle et technique entre le gouvernement de la République démocratique du Soudan et le gouvernement de la République française", signé à Khartoum le 22 décembre 1969, elle assure la coopération avec la direction des Antiquités du Soudan, tant dans le domaine des fouilles que dans celui de leur publication. La muséographie, la conservation du patrimoine et l'élaboration de rapports d'activité font également partie de ses missions.

La Sfdas est intégrée au service des Antiquités du Soudan (National Corporation for Antiquities and Museums, NCAM) au sein du musée national du Soudan (figure 10). Cette position privilégiée lui permet d'apporter un soutien aux missions françaises travaillant au Soudan et de développer des partenariats scientifique et muséologique. La Sfdas étant l'unique institut archéologique permanent au Soudan, elle occupe une position de fer de lance de la recherche scientifique française dans le pays. Au partenariat naturel avec la NCAM s'ajoutent des coopérations variées avec les établissements scientifiques français et les universités tant soudanaises qu'européennes. Avec l'appui de l'ambassade de France à Khartoum, elle permet à la diplomatie française d'entretenir durablement ses relations avec le Soudan.

La Sfdas a été dirigée successivement par André Vila (19691975), Francis Geus (1975-1984), Jacques Reinold (1984-2000), Francis Geus (2000-2004), Vincent Rondot (2005-2009), Claude Rilly (2009-2014) et, depuis septembre 2014, par Vincent Francigny. Chargée de coopérer 
avec la direction des Antiquités du Soudan dans ses activités de terrain (fouilles et prospections), elle a d'abord participé aux dernières opérations de sauvetage de la Campagne de Nubie qui précéda la mise en eau du lac de retenue du haut barrage d'Assouan.

Elle fut ensuite chargée de poursuivre le recensement systématique des sites de la vallée du Nil au sud de ce lac. Elle, a par ailleurs, effectué plusieurs fouilles programmées, notamment sur les sites de Missiminia (nécropole napatéenne, méroïtique, "groupe X" et chrétienne), el-Kadada (nécropole néolithique, méroïtique et post-méroïtique), Kadrouka (tertre funéraire néolithique) et el-Hobagi (tumuli post-méroïtiques).

\section{Prospections}

Pendant quatre ans, la Sfdas a participé, aux côtés de la NCAM, à un programme de prospections archéologiques et de fouilles de sauvetage lié à la construction d'un autre barrage dans la région de la quatrième cataracte du Nil. Ce programme a débuté en novembre 2001 et s'est achevé en 2005. La Sfdas a été chargée par la NCAM de mener quatre interventions dans les zones de repeuplement choisies pour l'installation des populations Chaggyia et Manassir qui habitent actuellement la quatrième cataracte. Ces régions se situent près de Debba, de Korti, d'Atbara et d'Abou Hamed. La première phase du programme, qui s'est terminée en décembre 2002, a concerné la région d'el-Multaga, à l'est de Debba. La prospection a permis l'identification de cent quarante-sept localités présentant un intérêt archéologique. Son apport concerne essentiellement la préhistoire, plus précisément des sites de débitage du Paléolithique moyen, des sites d'habitats contemporains du «Mésolithique» et du Néolithique de Khartoum, et des tertres funéraires datés de la seconde moitié du cinquième millénaire avant notre ère (figure 11).

Une seconde campagne de sauvetage s'est déroulée sur la rive droite du Wadi Muqaddam, de novembre 2003 à avril 2004. Cent quatrevingt-neuf sites ont été recensés. Le matériel le plus ancien recueilli à la surface témoigne d'occupations à la période acheuléenne et aux toutes premières phases du Paléolithique moyen. Comme ceux de Multaga, les sites néolithiques sont des habitats du «Mésolithique» de Khartoum et du Néolithique de Khartoum. La fouille de quatre cimetières post-méroïtiques de faible extension souligne l'installation de petites communautés en bordure des oueds et dans l'arrière-pays. Les sites chrétiens, les plus nombreux, sont en majorité des tombes isolées, parfois organisées en groupes de quelques unités.

La troisième région, au sud d'ed-Damer, a été prospectée d'octobre à décembre 2004. Sur une zone de $240 \mathrm{~km}^{2}$ (sud d'ed-Damer, site de Mukabrab), trente-cinq sites ont été inventoriés, la plupart sur d'anciennes 
terrasses du Nil situées à quelques kilomètres à l'est du cours actuel du fleuve. Le site le plus ancien est un habitat néolithique contemporain du «Mésolithique de Khartoum» et du "Néolithique de Khartoum». La découverte sur le site de tessons comparables à la céramique du Kerma ancien est une première dans cette région du Nil Moyen. Un petit cimetière de tombes méroïtiques et postméroïtiques sans matériel a fait l'objet d'une fouille de sauvetage, et quelques tombes tumulaires isolées d'époque chrétienne ont été étudiées.

La dernière opération, intitulée Kehaila East Archaeological Salvage Project, s'est déroulée en juin 2005 et a concerné la zone de Keheila entre Abou Hamed en aval et le village de Meheisa en amont. Cette zone désertique se caractérise principalement par des plaines à gravier traversées de petits ouadis, dont le plus important est le ouadi el-Go'oud. Les sites identifiés sont majoritairement néolithiques. Il faut également mentionner des sites «Kerma», «Post-Méroïtiques» et chrétiens.

\section{Fouilles archéologiques}

El-Hobagi ${ }^{81}$. Le site d'el-Hobagi, situé en rive gauche du Nil à $70 \mathrm{~km}$ en amont de Méroé, a été fouillé de 1985 à 1990 par une mission franco-soudanaise (Sfdas-NCAM) menée par Patrice Lenoble. Rien ne permettait de distinguer ces tumuli princiers d'époque post-méroïtique des centaines d'autres de la région. Sept sépultures ont été identifiées et deux fouillées. Un abondant matériel a été mis au jour (figure 12), similaire à celui découvert dans les pyramides les plus tardives de Méroé: vaisselle funéraire, armement, insignes de la royauté méroïtique (arcs, flèches, lances, anneaux d'archer, épées) et une abondante vaisselle en bronze gravée comportant un grand nombre d'éléments iconographiques méroïtique: uraeï, grenouilles, fleurs de lotus...

L'examen de ces sépultures comparées à l'iconographie des tombes (chapelles des pyramides) ou d'autres monuments royaux méroïtiques (temples, palais) nous donne d'importantes informations sur la caractérisation des rites royaux. Ainsi, l'abondance des armes retrouvées dans ces deux sépultures est à mettre en parallèle avec les scènes de l'iconographie royale méroïtique: massacres des prisonniers, scène de la soumission des Neuf Arcs (les pays soumis au pouvoir royal). La découverte du site d'el-Hobagi est capitale car elle démontre que cette région n'avait pas sombré, comme on l'avait longtemps présumé, après une hypothétique chute de Méroé. Une culture riche, imprégnée d'influences méroïtiques, a perduré pendant plusieurs siècles.

El-Kadada ${ }^{82}$. Découvert en 1976, el-Kadada est l'un des rares sites néolithiques connus dans la région de Shendi (figure 13). Les plus anciennes traces d'occupation sont des tessons de vase céramique dont le décor 


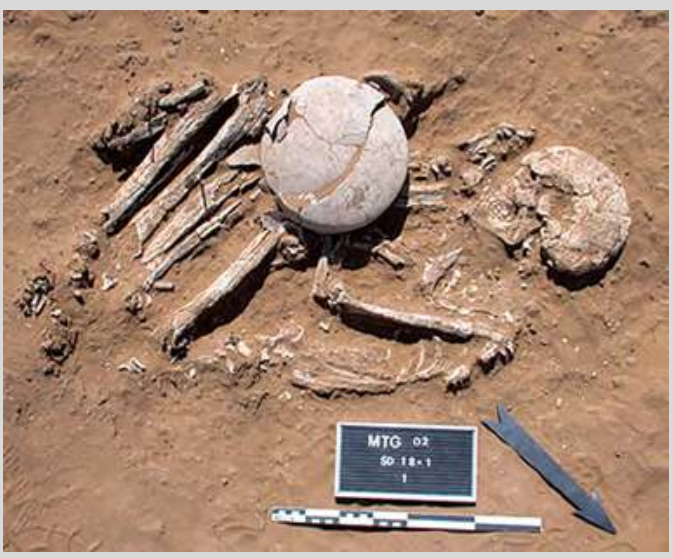

Figure 11: Sépulture d'el-Multaga.

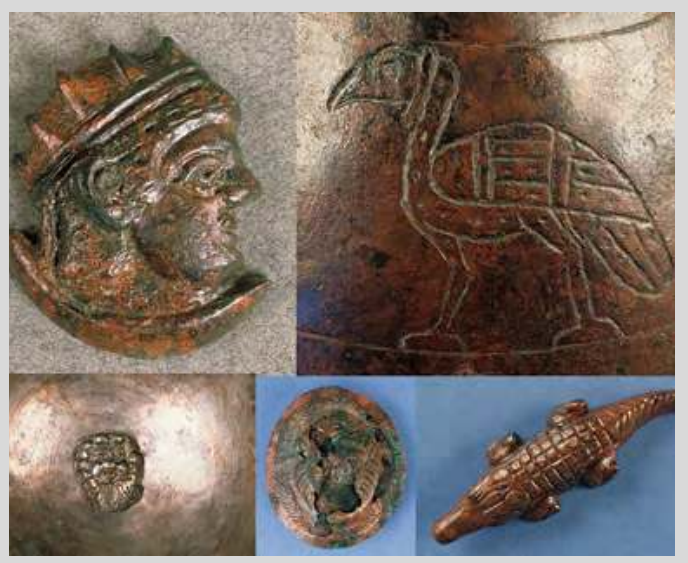

Figure 12: Collection royale d'el-Hobagi (voir p. 329).
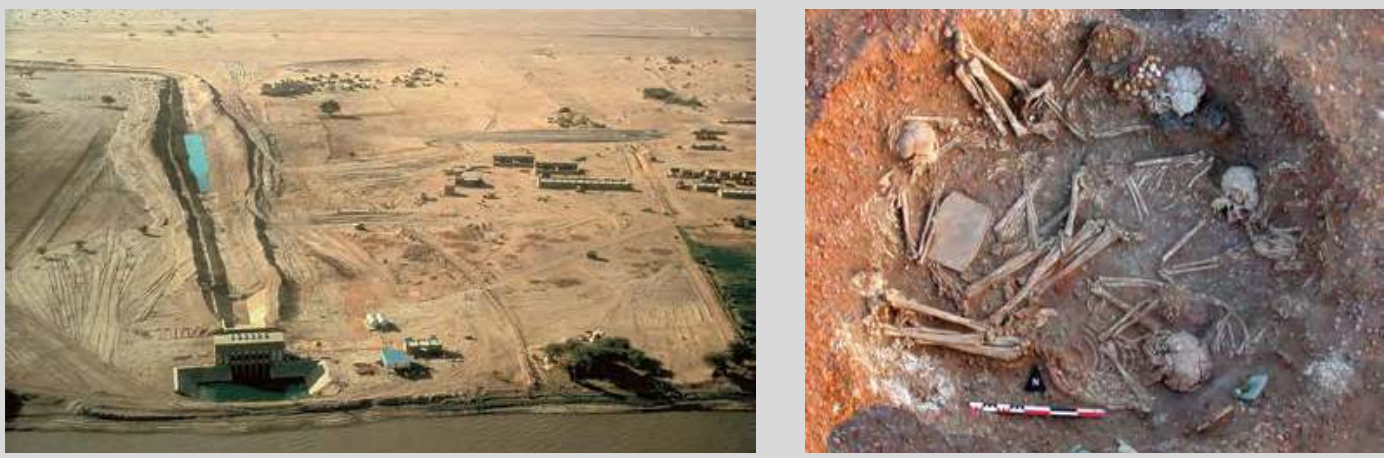

Figure 13: Site d'el-Kadada.

Figure 15: Sépulture néolithique d'el-Kadada KDD 86-141-144a.
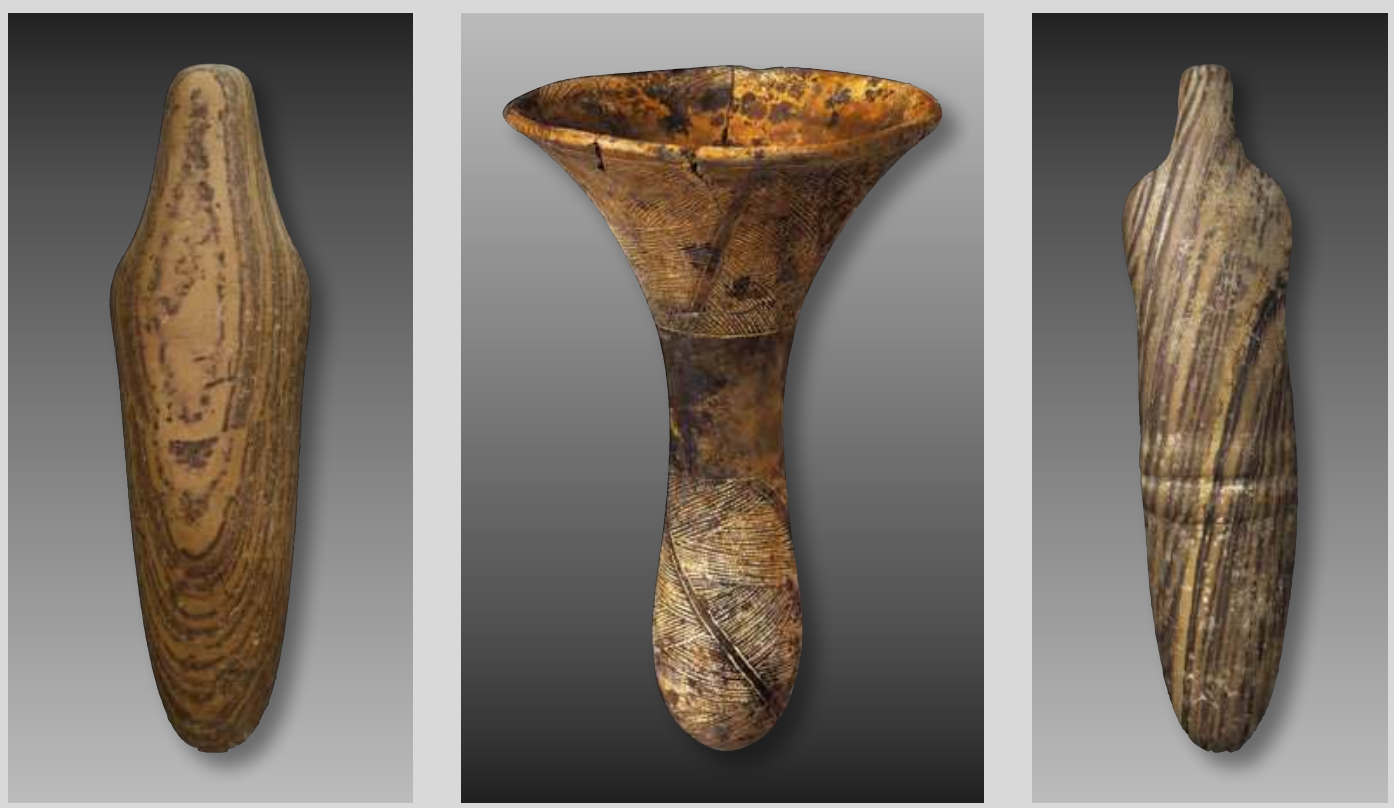

un siècle de fouilles

Figure 14: Figurines féminines SNM 26861 (KDK 1/131/8, voir p. 44) et 28731 (KDK 21/200/1) et vase caliciforme SNM 26883 (KDK 1/12/1) de Kadrouka. 
indique un peuplement au "Mésolithique de Khartoum», bien qu’aucun site de cette période n'ait été découvert. La période de transition suivante est attestée par des tessons identifiés sur un petit habitat, au lieu-dit el-Kudra, situé à moins de $200 \mathrm{~m}$. En revanche, aucune trace d'occupation à l'époque du "Néolithique de Khartoum», daté du cinquième millénaire avant notre ère, n'a été recensée. Il semble que les installations préhistoriques de cette période se soient déplacées vers le site d'el-Ghaba, $700 \mathrm{~m}$ plus au sud.

Le décor du matériel céramique trouvé dans les tombes d'el-Ghaba est comparable à celui des vases d'esh-Shaheinab, site éponyme du Néolithique de Khartoum. La découverte des tombes néolithiques d'el-Kadada est venue confirmer la suggestion de l'archéologue anglais A.J.Arkell, l'inventeur du Mésolithique et du Néolithique de Khartoum, d'un épisode préhistorique tardif. Les populations néolithiques d'el-Kadada ont développé à partir du quatrième millénaire une culture complexe qui se traduit dans le raffinement des décors céramiques et la nouveauté des formes, la finesse de l'outillage lithique et la grande richesse des dépôts funéraires.

El-Kadada recèle également un grand nombre de cimetières historiques, depuis la période napatéenne jusqu'au post-méroïtique, du huitième siècle avant notre ère jusqu'au sixième siècle après. Le matériel funéraire et le rituel des tombes pré-chrétiennes révèlent que la religion funéraire méroïtique ne s'achève pas avec la «fin de Méroé» mais avec le début de la christianisation, au $\mathrm{VI}^{\mathrm{e}}$ siècle de notre ère.

histoire et civilisations

Kadrouka $^{83}$. Le ouadi el-Khowi, qui correspond à d'anciens bras du Nil, se situe en rive est du fleuve, au sud de la troisième cataracte. Il est occupé par une grande densité de sites funéraires et d'habitats, parmi lesquels ceux du Néolithique tiennent une place importante. L'érosion éolienne a considérablement arasé les sites d'habitat qui ne se signalent plus aujourd'hui que par des épandages de matériel en surface. Les cimetières, en revanche, installés sur des buttes, ont bien résisté, et leur fouille est aujourd'hui le moyen privilégié pour comprendre les sociétés habitant la région entre les $\mathrm{VI}^{\mathrm{e}}$ et $\mathrm{IV}^{\mathrm{e}}$ millénaires (figure 14).

Les interventions de sauvetages menées par la Sfdas - la région est en effet depuis plusieurs années touchée par des programmes de développement agricole — ont identifié une cinquantaine de ces sites néolithiques, funéraires ou d'habitat. Sur la vingtaine de cimetières connus, six ont été fouillés exhaustivement ou partiellement par la Sfdas. À l'exception de deux tertres funéraires qui doivent dépasser le millier de tombes, les autres renferment un peu plus d'une centaine d'inhumations. Près de 700 sépultures, dont les dates sont incluses entre 4800 et 4000 avant notre ère, 
ont pu être enregistrées. Les constantes et les variantes observées dans les domaines de la culture matérielle et des coutumes funéraires paraissent traduire à la fois l'homogénéité de ces populations et une évolution rapide de leur tissu social. Ces cimetières témoignent d'une organisation où la hiérarchisation se fait de plus en plus forte. Au cours du Néolithique, des chefferies se mettent en place avec l'émergence d'un personnage dominant. Ces sociétés vont constituer les bases des premiers états protohistoriques, préludant ainsi à l'apparition des premiers royaumes.

Autres fouilles françaises. Parmi les autres fouilles françaises en activité au Soudan, citons Sedeinga ${ }^{84}$, situé entre la seconde et la troisième cataracte, sur la rive gauche du Nil. Le site se trouve à une trentaine de kilomètres au sud de l'île de Saï. Il a d'abord été connu pour les vestiges du temple de la reine Tiyi, la grande épouse d'Amenhotep III. Cette ruine, avec son unique colonne encore debout, est trop fragile pour que des fouilles puissent y être effectuées sans une restauration des blocs de grès pulvérulents qui la composent. Entre le temple et le désert s'étend, sur près de 40 ha, une immense nécropole napatéenne et méroïtique, la plus vaste actuellement connue et préservée en Nubie.

À quelques kilomètres au sud de la cataracte de Dal et du lac de Nubie qui recouvre désormais la Basse Nubie, l'île de Saï ${ }^{85}$ est un véritable conservatoire archéologique naturellement protégé du développement humain gagnant les berges de la vallée nubienne. Comptant parmi les plus grandes îles jalonnant le Nil, elle héberge des vestiges qui s'étendent du paléolithique jusqu'aux époques modernes, et fait l'objet de campagnes de fouilles régulières. Enjeu territorial majeur entre les royaumes nubiens et l'Empire égyptien, elle a notamment conservé les traces d'une ville pharaonique enfouie pour un tiers sous les murs d'une forteresse d'époque ottomane. Recouverte dans sa partie nord par d'immenses champs tumulaires post-méroïtiques, l'île possède également des nécropoles appartenant au Nouvel Empire ainsi qu'aux époques «Kerma», napatéenne, méroïtique et chrétienne.

Grâce aux activités menées sur l'ensemble des sites mentionnés, et aux recherches conduites par l'archéologie française et internationale pendant près de soixante ans, le patrimoine soudanais a pu obtenir la reconnaissance internationale qu'il mérite. La variété des approches listées - combinée aux collaborations internationales toujours plus nombreuses - permet de valoriser et d'enrichir cette discipline, certes encore jeune, qu'est la nubiologie. À un moment où l'archéologie de terrain tient une part très importante, et où la formation des chercheurs spécialisés 
dans le domaine est prolifique, le rôle de la Sfdas dépasse aujourd'hui largement ses prérogatives initiales. Insistons ici sur le rôle fondamental qu'elle joue grâce à la formation des archéologues français et soudanais dans la protection et la valorisation du patrimoine soudanais.

Grâce aux deux expositions qui se sont tenues en 1997 à l'institut du Monde arabe — «Soudan, royaumes sur le Nil» ${ }^{86}$ — puis en 2010 au musée du Louvre — «Méroé, un empire sur le Nil » ${ }^{87}$-, le grand public est aujourd'hui plus au fait de la réalité de la recherche scientifique au Soudan et fait montre d'un appétit grandissant pour ce pays et son histoire. Cependant, des territoires scientifiques entiers sont encore méconnus et demeurent à "conquérir»: la région du Kordofan, si importante pour comprendre les relations entretenues avec l'Afrique centrale, ou encore la zone située au sud de la ville de Khartoum sont de bons exemples. Pourtant, qu'il s'agisse de fouilles programmées ou de prospections, les résultats obtenus en un peu plus d'un demi-siècle sont considérables. La découverte à Doukki Gel, près de l'ancienne capitale Kerma, d'une cache contenant les statues brisées des souverains de Napata, ou encore, dans le domaine néolithique, la mise au jour d'une tombe à sacrifice humain (ou mort d'accompagnement) à el-Kadada sont, de ce point de vue, révélatrices (figure 15).

Avec le prochain chapitre dédié à l'urbanisme méroïtique, nous verrons que, malgré les progrès exponentiels réalisés par la discipline archéologique au Soudan, les Joyeux n'en sont encore qu'au début des choses

histoire et 


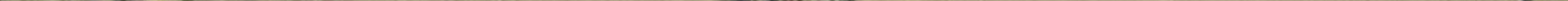




\section{notes}

1 Ahmed 2010, 29-34.

2 Welsby 1996.

3 Edwards 2004.

4 Mainterot 2010b, 22-27; 2011.

5 Shinnie 1967, 24-27; Török 1997, 7-20.

6 Mainterot 2010a, 19-21.

7 Burckhardt 1813, 275. Notons qu'il n'identifia pas le site à la ville de Méroé.

8 Chauvet 1989; Dewachter 1994.

9 Leclant 1967, 6-15; Chauvet 1989.

10 Caillaud 1826.

11 Shinnie 1958, 75, 138-150.

12 Ferlini 1837.

13 Freier et Reinecke 1984; Freier et Grunert 1996.

14 Lepsius 1849-1859.

15 Budge 1907.

16 Griffith 1911c et 1912; Crowfoot 1920, 85-92.

17 Scott-Moncrief 1908, 192-203.

18 Garstang 1911; Garstang 1914, 9-23; Garstang 1916, 1-27.

19 Reisner 1910; Firth 1912, 1915, 1927.

20 Woolley 1911.

21 Woolley et MacIver 1910.

22 Griffith 1924, 141-180;

Griffith 1925, 57-172.

23 Crawford et Addison 1951.

24 Addison 1949; Addison 1956, 4-18.

histoire et

civilisations
25 Dunham 1950, 1955.

26 Dunham 1970.

27 Dunham 1957.

28 Dunham 1963.

29 Reisner 1923, 34-77.

30 Crowfoot 1920, 85-92; Addison 1926, 56-58; Addison et Dunham 1922, 39-46; Whitehead 1928, 59-67.

31 Emery et Kirwan 1935.

32 Emery et Kirwan 1935, 108.

33 Kirwan 1936, 200-211; Macadam 1949, 1955.

34 Smith et Adam 1950, 301-306.

35 Balfourpaul 1952, 202-215.

36 Hintze 1959, 171-196.

37 Hintze 1962, 170-202; Hintze 1963, 217-226; Hintze 1968, 667-684.

38 Vercoutter 1962, 263-299.

39 Shinnie 1970.17-19; Shinnie 1984, 498-504; Shinnie et Bradley 1980.

40 Smith 1962; Adams et Nordström, 1963, 10-46; Klasens 1967, 79-86.
41 Caminos 1968.

42 Leclant 1962, 197-222;

Leclant 1963, 17-25.

43 Millet 1963, 147-165, pl. XLV-XLVII;

Millet 1964, 7- 14, pl. I-VIII ; Millet 1967, 123-126; Millet 1968.

44 Jacquet 1971, 121-131.

45 Trigger 1967.

46 Verwers 1962, 19-33; Hewes 1964, 174-187.

47 Verwers 1962, 19-33.

48 Adams 1965, 148-176.

49 Adams et Nordström 1963, 10-46.

50 Emery 1965; Trigger 1976.

51 Adams 1977.

52 Maystre 1973, 193-199; Jacquet-Gordon, Bonnet, Jacquet 1969; Jacquet-Gordon, Bonnet 1999.

53 Welsby 1996; Török 1997b.

54 Mallinson 1996.

55 Paner 1997, 137-155.

56 Zurawski 2002, 73-85.

57 Eisa 1999; Welsby 2001a; Welsby 2003.

58 Edwards 1998.

59 Les différents instituts ayant participé au projet comprennent: l'université de Cologne et son projet Acacia (survey de l'île de Boni), le musée archéologique de Gdańsk et son projet Game (Gdanisk Archaeological Museum Expedition), la Polish Academy of Sciences, la Humboldt University of Berlin et son projet Hune (Humboldt University Nubian Expedition), l'IsiAo (Italian Institute for Africa and the Orient), l'UcL Institute of Archaeology, la SARS (Sudan Archaeological Research Society) et son projet Amri to Kirbekan Survey 1999-2007 (nombreuses attestations d'occupations du Méroïtique tardif et Post-Méroïtique), la Hungarian Meroe Foundation, l'Uсsв (University of California at Santa Barbara) et l'Asu (Arizona State University) et leur projet commun (Ucsi-Asu Fourth Cataract Archaeological Expedition) ayant pour nom The UCSB West Bank Archaeological Survey from el Kab to Mograt, (aucun site méroïtique recensé) et l'Oriental Institute Museum of the University of Chicago et son projet The Oriental Institute Nubian Expédition (aucun site méroïtique recensé). 
60 Pour une revue exhaustive de la bibliographie du survey, voir la page internet http://www. nubiansociety.org/mdasp_articles.html.

61 Welsby 2003, 5-7; Payne 2007, p. 9-13; Ginns 2006, 18 et Thomas 2008, 64-66.

62 Bonnet et Ahmed 1999, 251-256; Bonnet et Valbelle 2004b, 109-112, Bonnet et Valbelle 2010, 43-50.

63 Welsby 2000, 5-10; Welsby 2001b, 64-70; Welsby 2002, 26-39; Welsby 2004b, 148-157; Welsby 2009, 72-77; Welsby 2010, 48-55.

64 Donadoni 1993, 101-115; Donadoni 1994, 54-59; Roccati 1997, 12-18; Roccati et Bergamini 1999, 633-642; Roccati 2003, 59-64; Roccati 2004b, 384-388;

Roccati 2008, 249-261.

65 Kendall 1991, 302-313; Kendall 1994, 139-144; Kendall 1997a, 161-228; Kendall 1997b, 320-354; Kendall et Wolf 2007, 82-88; Kendall et Wolf 2011, 237-26o.

66 Ahmed et Anderson 2000, 17-37, Ahmed et Anderson 2005, 10-27; Ahmed et Anderson 2006, 2-3; Ahmed et Anderson 2008, 40-46; Ahmed et Anderson 2010, 50-55.

67 Grzymski 2006, 227-238; Grzymski 2008, 47-51.

68 Wolf, Hof, Onasch 2003, 71-87 et 2008, 101-116; Wolf S., Wolf P., Onasch, Hof et Nowotnick 2008, 157-230 et 2009, 215-262.

69 Wolf P. 2002, 92-111; 2004c, 83-97; 2006, 257-264.

70 Paner 2005, 54-55; El-Tayeb et Kolosowska 2005, 145-158.

71 Lenoble et Rondot 2003, 101-115; Rondot 2005, 399-401;

Rondot 2006, 41-47.

72 Lenoble et Sokari 2005, 59-61;

Baud 2008, 52-53; Baud 2010d, 218-224;

Baud 2011, 339-357.

73 Wolf 1997, 20-29; Wolf 1998, 10-12;

Wenig et Wolf 1998-2000;

Wolf 2001a et b; Wolf 2004a, 436-445;

Wolf 2004d, 47-101; Näser 2011, 317-338.

74 Wildung 1998, 183-190; Wildung 1999;

Wildung et Kroeper 2006 et 2011 ;

Kroeper 2006, 287-296.

75 Ahmed et Anderson 2003, 25-34.

76 Gradel 2009, 387.

77 Vercoutter et Adam 1961.
78 Pour une rétrospective dédiée à l'action menée par l'université Charles-deGaulle-Lille 3 au Soudan, on consultera Gradel 2009, 383-400.

79 Gradel 2009, 389-390.

80 La Sfdas est l'un des 27 instituts français de recherches à l'Étranger (IFRE), financés par le ministère des Affaires étrangères et du Développement international: www.sfdas.com; http://www.ambafrance-sd.org/ SCAC-service-de-cooperation-et-d-293).

81 Lenoble 1997, 289-308; Lenoble 1999, 157-197; Lenoble 2004, 193-195.

82 Reinold 1986, 159-169; Reinold 1994, 51-66;

Reinold 2008; Lenoble 1987, 89-119.

83 Reinold, 1991, 16-29; Reinold 1998, 19-40;

Reinold 2000.

84 Rilly et Francigny 2010, 62-68; Rilly et Francigny 2011, 72-79.

85 Francigny 2010a, 62-67; 2010b, 56-61; 2015, 201-212.

86 Wildung 1997.

87 Baud 2010a. 